\title{
Saturación en los servicios de urgencias: Análisis de cuatro hospitales de Medellín y simulación de estrategias*
}

\author{
Emergency Department Overcrowding : A Four-Hospital Analysis in Medellín and a Strategy Simulation \\ Saturação nos serviços de urgência: Análise de quatro hospitais de Medellín e simulação de estratégias
}

Jairo Humberto Restrepo-Zea a

DOI: https://doi.org/10.11144/Javeriana.rgps17-34.ssua

Universidad de Antioquia, Colombia

ORCID: http://orcid.org/0000-0002-3449-0373

Fecha de recepción: 22 Marzo 2017

Juan Sebastián Jaén-Posada

Universidad de Antioquia, Colombia

Fecha de aceptación: 20 Octubre 2017

ORCID: http://orcid.org/0000-0001-7077-5109

Juan José Espinal Piedrahita

Universidad de Antioquia, Colombia

Paula Andrea Zapata Flórez

Universidad de Antioquia, Colombia

\section{Resumen:}

Las urgencias médicas pueden saturarse debido a factores externos e internos al servicio. Esto se refleja en congestión, tiempos de espera elevados e insatisfacción de los usuarios. Identificar y simular estrategias para gestionar las urgencias médicas, procurando atenuar la saturación. Investigación exploratoria y analítica, con trabajo de campo en cuatro unidades de urgencias de Medellín y simulación de estrategias contra la saturación mediante dinámica de sistemas. Las estrategias utilizadas son: reorganizar el registro, remitir los pacientes que no requieren atención urgente, implementar consultorio fast-track e incrementar el traslado a pisos. Esta última es la estrategia más efectiva en la simulación. Aunque las estrategias de flujo pueden ayudar a resolver la saturación a partir de sus causas internas, como lo sugiere la literatura, su alcance es limitado. Se requieren estrategias de entrada, que no están al alcance del servicio, y estrategias de salida del sistema hospitalario. Es posible disminuir la saturación, concibiendo las urgencias y el hospital como dos elementos de un mismo sistema, de modo que se agilice el flujo de pacientes y se cuente con oferta adecuada de camas hospitalarias sin sobredimensionar la capacidad instalada.

Palabras clave: urgencias médicas, triage, acceso, saturación, eficiencia hospitalaria, dinámica de sistemas.

\section{Abstract:}

Medical emergency services can be overcrowded due to both external and internal service factors. This is evinced in the congestion, longer waits, and dissatisfaction by the users. To identify and simulate strategies for processing medical emergency requests in order to mitigate the overcrowding. An exploratory and analytical research was conducted based on the field work at four emergency rooms in hospitals of Medellín; simulation strategies were then proposed against the overcrowded service with system dynamics. The strategies included: to reorganize the sign-in, refer to other places those patient not requiring emergency services, implement fast-track services, and increase the inpatient capacity. The last one becomes the most effective strategy in the simulation. While the flow strategies can help to solve the service overcrowding due to internal causes as suggested in the literature, these strategies are limited in their scope. The required patient sign-in strategies as well as the patient discharge strategies are not easy-to-do in these emergency rooms. Overcrowding can be reduced by thinking of the hospital and the emergency room as two elements in a single system, so that the patient flow can be speeded up; the bed number for inpatients must be improved without overflowing the hospital capacity.

Keywords: medical emergencies, triage, access, overcrowding, hospital efficiency, system dynamics.

\footnotetext{
a Autor de correspondencia. Correo electrónico: jairo.restrepo@udea.edu.co
} 


\section{Resumo:}

As urgências médicas podem se saturar devido a fatores externos e internos ao serviço. Isso se reflete em congestão, tempos de espera elevados e insatisfação dos usuários. Identificar e simular estratégias para gerir as urgências médicas, procurando atenuar a saturação. Pesquisa exploratória e analítica, com trabalho de campo em quatro unidades de urgências de Medellín e simulação de estratégias contra a saturação mediante dinâmica de sistemas. As estratégias utilizadas são: reorganizar o cadastro, reencaminhar os pacientes que não precisam atendimento urgente, implementar clínica ambulatória fast-track e facilitar o traslado aos quartos. Esta última é a estratégia mais efetiva na simulação. Ainda que as estratégias de fluxo possam ajudar a resolver a saturação a partir de suas causas internas, como sugere a literatura, seu escopo é limitado. Precisam-se estratégias de entrada, que no estão dentro do escopo do serviço e estratégias de saída do sistema hospitalar. Conclusões: é possível diminuir a saturação, concebendo as urgências e o hospital como dois elementos de um mesmo sistema, de jeito de agilitar o fluxo de pacientes e contar com oferta adequada de leitos hospitalares sem superdimensionar a capacidade instalada.

Palavras-chave: urgências médicas, triagem, acesso, saturação, eficiência hospitalar, dinâmica de sistemas.

\section{Introducción}

Las urgencias médicas constituyen un caso especial del acceso a servicios de salud. En primer lugar, en estos servicios se atienden necesidades asociadas con deterioros del estado de salud, en casos extremos cuando se pone en riesgo la vida de las personas. Quienes buscan estos servicios padecen una dolencia o accidente cuya atención debe ser inmediata o prioritaria, sin importar su costo y dando prioridad a la expectativa de vida. Precisamente, como podrían darse barreras financieras para el acceso, los sistemas de salud establecen la atención obligatoria y gratuita, y entre las opciones de financiación se encuentran, además de los seguros tradicionales de salud, otros seguros especiales como los destinados a cubrir la atención de víctimas de accidentes de tránsito o de accidentes de trabajo $[1,2]$.

En segundo lugar, las urgencias constituyen una puerta de entrada a los hospitales, bien porque desde allí se dispone la hospitalización de pacientes, o bien porque es necesario acudir a cirugía para atender una emergencia médica. En una revisión sistemática realizada en 2015 se encontró que en 59 países de ingresos bajos y medios el 55.7\% de los ingresos hospitalarios se realizó por medio de los servicios de urgencias [3]. En este sentido, los servicios de urgencias son interdependientes del sistema hospitalario, tanto en relación con el hospital del que hacen parte como con las redes locales o regionales que los rodean [4].

Ahora bien, la demanda por urgencias puede estar influenciada no solamente por hechos aleatorios que ponen en riesgo la vida de las personas - como un accidente de tránsito, algún tipo de agresión externa o un infarto-, sino también por las condiciones de acceso en otros servicios, por las expectativas de los usuarios o por la normatividad y las barreras legales que puedan afectar el servicio. Un caso especial se refiere a la demanda que se expresa en urgencias como resultado de la insatisfacción de los usuarios que no encuentran atención oportuna en consulta médica, lo que incluso puede significar que dadas las patologías subyacentes se dé lugar a una desestabilización o descompensación de los pacientes $[5,6]$.

$\mathrm{Al}$ enfrentar un posible desequilibrio entre la oferta y la demanda, parece inevitable que se presente una saturación de las urgencias. Este fenómeno se ve reflejado principalmente en largas esperas, en la ocupación de las áreas comunes, la utilización de sillas, mesas e incluso el suelo para la atención de los pacientes, en el abandono del servicio y en el alto nivel de estrés del personal asistencial, entre otros [7]. Entre las escalas de medición sobre la saturación se destacan el Emergency Department Word Indexe (EDWIN), el Emergency Department Crowding Score [7] y el tiempo de atención o duración de la estancia [8]. También se cuenta con el modelo de medición National Emergency Department Overcrowding Score (NEDOCS), el cual fue aplicado en un estudio en tres hospitales de Colombia [9].

En cuanto a las causas de la saturación, estas se pueden atribuir a múltiples factores [10] y se agrupan en causas externas o internas, en la medida en que provengan de fenómenos por fuera del servicio o que se trate de características o limitaciones propias de este. En diversos estudios se plantean tres categorías más específicas de las causas — de entrada, de caudal o flujo y de salida - , las cuales permiten plantear soluciones al problema de saturación. Según Asplin [4], las soluciones relacionadas con la entrada intentan influenciar la demanda 
y parecen virtualmente imposibles, excepto que se puedan impactar los factores de riesgo que dan lugar a las emergencias, o bien que el sistema de salud ofrezca oportunidad y calidad en la atención de los servicios ambulatorios. En este sentido, para reducir o contener la demanda se busca intervenir los procesos previos a las urgencias, como la consulta externa, buscando que el estado de los pacientes permanezca estabilizado o en mejoría. Las políticas de ingreso propias también pueden influir, lo que puede dar lugar a discriminación [11] y a negar el acceso a quienes ya han intentado acceder mediante consulta médica, y no contribuyen a una solución efectiva de la saturación [7].

Las soluciones de caudal o de flujo se tienen dentro del servicio y corresponden a estrategias de gestión de procesos que se concentran en el tiempo de estancia y buscan mejorar la eficiencia del servicio, intervienen el triage, la asignación de habitaciones, la realización de exámenes y el diagnóstico. Dentro de estas soluciones se encuentran el fast track, el streaming, el point-of-care testing y el bedside registration $[5,12,13]$.

Finalmente, las estrategias de salida están determinadas por la facilidad con que los usuarios pueden pasar a un proceso posterior a las urgencias. Un ejemplo de ello es la preparación de camas de hospitalización para los pacientes que ya fueron admitidos, o el traslado de pacientes a hospitalización para esperar por cama y despejar el área de urgencias. Es evidente que la carencia de camas en hospitalización ocasiona que los pacientes permanezcan en urgencias y obstaculicen el ingreso de nuevos pacientes al servicio; además, exigen mayor disposición de médicos y enfermeras para revisar su estado de salud [4], así que la mayor capacidad en hospitalización agilizaría el flujo y reduciría la saturación. Sin embargo, esta aceleración podría inducir la demanda en urgencias, en la medida en que las personas asistirán al servicio porque verán solucionada su condición en un menor tiempo que en una consulta médica general, y con ello el resultado en cuanto a saturación podría seguir siendo negativo.

En el caso colombiano, las instituciones prestadoras de servicios de salud (IPS) — clínicas y hospitalesconstituyen un subsistema base del sistema de salud, pues son las responsables de brindar la atención a la población. Las políticas que se adopten en el sistema influyen directa o indirectamente en el funcionamiento de estas, y es así como los servicios de urgencias constituyen unidades clave. En estos servicios se reflejan las políticas de cobertura universal, en particular la obligación que adquieren de prestar la atención de urgencias con independencia de la capacidad económica de los usuarios.

Aunque no se tienen muchos estudios publicados para el caso colombiano, es muy común en los medios de comunicación y en los debates sobre el sistema de salud la referencia a la saturación de las urgencias médicas. Algunos estudios reconocen que el problema de acceso al servicio podría ser resultado de la saturación [14]. Del mismo modo, se ha enunciado el marco que rodea a la saturación y se consideran los cambios jurídicos por los que el país ha atravesado, con referencia a las posibles causas externas de la saturación y a las soluciones a partir de ellas [15]. Desde una mirada pública, la prensa denuncia con frecuencia la saturación existente y la aparente poca capacidad de la infraestructura hospitalaria. En la prensa nacional se reconoce el problema creciente para los usuarios desde la perspectiva no solo de los pacientes, sino también de las instituciones de salud $[16,17,18,19,20,21]$. La saturación es atribuida a causas externas e internas, pero en general se observa una creciente congestión ante la falta de recursos para sobrellevarla.

En tal contexto, en este artículo se propone un marco conceptual para el análisis y la gestión de los servicios de urgencias, con una aproximación empírica en cuatro hospitales de alto nivel de complejidad de la ciudad de Medellín y un ejercicio de simulación de estrategias para hacer frente a la saturación, de modo que se conozca cuáles pueden ser más eficaces para reducir los tiempos de espera y la estancia. Los resultados del análisis, a partir de la experiencia de los cuatro hospitales y del ejercicio de simulación para un hospital característico, permitirán plantear alternativas para la gestión hospitalaria y para la discusión pública sobre la situación que presentan estos servicios. 


\section{Metodología y análisis de datos}

Se realizó un estudio exploratorio y analítico. Exploratorio, por cuanto se abordó el tema de la saturación de las unidades de urgencias, tratando de identificar las estrategias para su mitigación en la red de servicios de la ciudad de Medellín. Y analítico, dado el propósito de plantear un marco conceptual y una simulación de sistemas para representar el flujo del servicio y establecer los resultados que pueden presentarse al adoptar las estrategias identificadas en la fase exploratoria.

\section{Modelo conceptual}

El objeto de estudio es el servicio de urgencias médicas, el cual fue concebido y representado mediante un esquema con los diferentes momentos de atención —entrada o búsqueda del servicio, admisión, triage, atención y egreso-, los tiempos de espera —en admisión, triage y atención- y los abandonos que se producen en este contexto (figura 1). El esquema muestra el flujo que un paciente recorre generalmente en el servicio, desde la entrada o búsqueda hasta que se resuelve su situación. El paciente puede provenir de alguna de cinco posibles situaciones o lugares donde percibió síntomas de enfermedad o sufrió algún accidente: de su hogar, de una institución educativa; del trabajo; de algún sitio público, o desde otro establecimiento hospitalario del cual es remitido.

En una primera etapa, el paciente encuentra al personal de seguridad en la entrada, que en muchos casos le indicará a dónde debe dirigirse o, en el caso de un paciente en estado crítico, permite su paso inmediato para la atención inicial de su padecimiento. En la segunda etapa, se encuentra la admisión, donde se registra la entrada del paciente, generalmente se recibe documentación e información básica. La tercera etapa, el triage, clasifica al paciente según criterios de gravedad clínica, para lo cual se siguen varios modelos de clasificación, como el australiano que emplea cuatro niveles y el de Manchester con cinco niveles [22, 23], desde un triage uno que significa riesgo vital inmediato o alto riesgo hasta los triage superiores que indican urgencias de riesgo vital moderado a bajo. Después de la clasificación, el paciente recibe la atención que, de acuerdo con su gravedad, puede ser más rápida o presentarse como "cita prioritaria", donde se atienden dolencias de menor gravedad pero que requieren una pronta atención.

A partir de la atención, el paciente tiene varios canales de salida: es dado de alta, es admitido en hospitalización, se transfiere a otro centro hospitalario, es ingresado a cirugía o fallece. Como se aprecia en la figura 1, en ciertas etapas de este flujo se presentan tiempos de espera, como en la admisión, el triage, la atención o el egreso, donde pueden generarse colas, que de ser muy extensas pueden dar lugar a abandonos y a la insatisfacción del paciente. En este esquema general de urgencias las esperas se generan entre cada etapa del paciente, y allí es donde se identifican los momentos de saturación y se pueden adoptar estrategias de solución. 


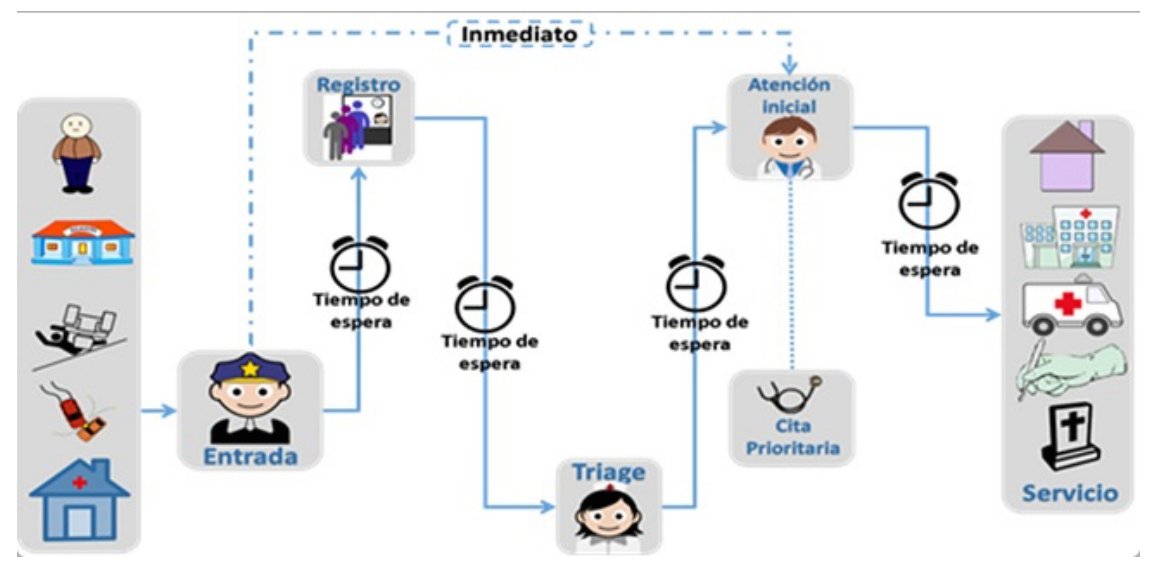

FIGURA 1

Flujo del paciente en los servicios de urgencias médicas

Fuente: elaboración propia.

\section{Trabajo de campo: información de cuatro hospitales}

Se llevaron a cabo visitas a cuatro unidades de urgencias de Medellín ${ }^{1}$, durante el mes de julio de 2014, en las cuales se buscó validar el esquema planteado (figura 1), recolectar información para el análisis sobre la magnitud de la saturación, identificar los puntos críticos que podrían generarla en cada etapa del proceso y conocer las estrategias para enfrentarla. Se desarrolló un cuestionario semiestructurado como guía para las entrevistas con los coordinadores de cada unidad de urgencias. También se diseñó una ficha para recolectar información estadística sobre la capacidad instalada del proceso de atención, información relativa a la prestación del servicio sobre los pacientes remitidos a otras unidades de menor complejidad, el número de personas que abandonan el servicio debido a la saturación y la oportunidad de la atención basada en los tiempos promedios de cada etapa. La ficha también contenía información respecto a la calidad percibida y a la satisfacción de los pacientes, la cual se capta mediante mecanismos de recepción de quejas, reclamos y peticiones de las instituciones (tabla 1). 
TABLA 1

Información para el análisis de las unidades de urgencias médicas

\begin{tabular}{|c|c|c|}
\hline Criterios & Variables & Indicadores \\
\hline $\begin{array}{l}\text { Capacidad } \\
\text { instalada }\end{array}$ & $\begin{array}{l}\text { - Número de taquillas para registro } \\
\text { - Número de consultorios para triage } \\
\text { - Personal para registro, triage y } \\
\text { consulta } \\
\text { - Número de camas, camillas y sillas } \\
\text { - Número de personal en urgencias } \\
\text { - Espacio para expansión }\end{array}$ & $\begin{array}{l}\text { - Número de personas ingresan a } \\
\text { urgencia } \\
\text { - Número de personas que ingresan a } \\
\text { triage } \\
\text { - Número de personas que ingresan a } \\
\text { consultas } \\
\text { - Número de hospitalizados }\end{array}$ \\
\hline $\begin{array}{l}\text { Prestación de } \\
\text { servicios }\end{array}$ & $\begin{array}{l}\text { - Tiempo promedio desde el registro } \\
\text { hasta el triage } \\
\text { - Tiempo promedio desde el triage } \\
\text { hasta la consulta } \\
\text { - Tiempo promedio desde el servicio } \\
\text { inicial hasta la hospitalización } \\
\text { - Promedio de personas que son } \\
\text { remitidos a otra institución después } \\
\text { del triage personas que } \\
\text { - Número de por saturación }\end{array}$ & $\begin{array}{l}\text { - Oportunidad en la atención en } \\
\text { consulta de urgencias } \\
\text { - Duración promedio de la estancia en } \\
\text { urgencias } \\
\text { - Oportunidad en la atención por triage } \\
\text { - Promedio de personas que } \\
\text { abandonan el servicio de urgencias } \\
\text { - Promedio de personas que se remiten } \\
\text { a otras instituciones }\end{array}$ \\
\hline $\begin{array}{l}\text { Calidad y } \\
\text { satisfacción de } \\
\text { los servicios }\end{array}$ & $\begin{array}{l}\text { - Mecanismos de recepción de } \\
\text { peticiones, quejas y reclamos } \\
\text { - Promedio de sugerencias en la } \\
\text { atención de urgencias } \\
\text { - Promedio de quejas y reclamos por } \\
\text { los servicios de urgencias } \\
\text { - Promedio de peticiones por } \\
\text { servicios }\end{array}$ & $\begin{array}{l}\text { - Proporción de quejas resueltas antes } \\
\text { de quince dias en urgencias } \\
\text { - Tasa de satisfacción global en } \\
\text { urgencias }\end{array}$ \\
\hline
\end{tabular}

Fuente: elaboración propia a partir de la guía de entrevista para el personal de los servicios de urgencias médicas en la ciudad de Medellín.

\section{Simulación del sistema de urgencias: análisis de datos de hospital característico}

A partir de la información de las cuatro unidades visitadas, se propuso simular en un hospital típico o característico de alta complejidad las estrategias implementadas en las instituciones con mayor grado de saturación. Las simulaciones se realizaron de acuerdo con la metodología conocida como dinámica de sistemas (DS), una herramienta que permite el modelamiento de sistemas para una representación realista de los procesos de una forma más agregada y costoeficiente en términos de los inputs que requiere el modelo de simulación [24]. La DS como ninguna otra metodología de simulación se enfoca en evidenciar los aspectos estructurales del sistema que explican el comportamiento observado. El modelamiento se efectúa mediante la representación de las variables del sistema en términos de flujos y niveles, como se muestra en la figura 2 en la que se denotan los flujos como válvulas y los niveles como tanques. Los flujos representan las razones de 
cambio de las variables en función del tiempo, como el número de pacientes que llegan al servicio, el número de pacientes atendidos y los pacientes trasladados a pisos. En cuanto a la unidad de tiempo para representar el flujo, es elegida por el modelador de acuerdo con el nivel de detalle que desee representar el sistema. En este caso, se obtuvo la información de los pacientes por hora.

En cuanto a los niveles o acumulaciones, estos surgen de la diferencia entre los flujos de entrada y los de salida de un proceso. Por ejemplo, si el número de pacientes que demandan el servicio por hora es mayor que el número de pacientes atendidos por hora surge un nivel entre estos dos flujos que se llama pacientes en espera o pacientes en cola.

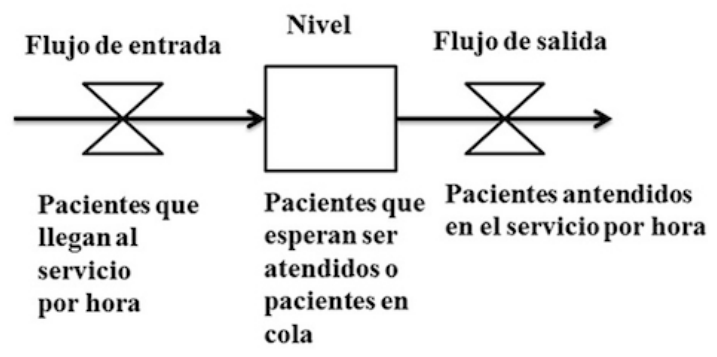

FIGURA 2

Representación de un sistema de atención de pacientes simple Fuente: elaboración propia.

La modelación se hizo mediante el uso del programa computacional Powersim Studio 10, a partir de una unidad de urgencias típica en la que se consideran básicamente cuatro aspectos: la llegada al servicio, el registro, la atención (que puede incluir algún tipo de tratamiento) y el traslado a piso o alta. En la figura 3 se presenta una versión simplificada de la unidad de urgencias típica sobre la cual se simularon las estrategias.

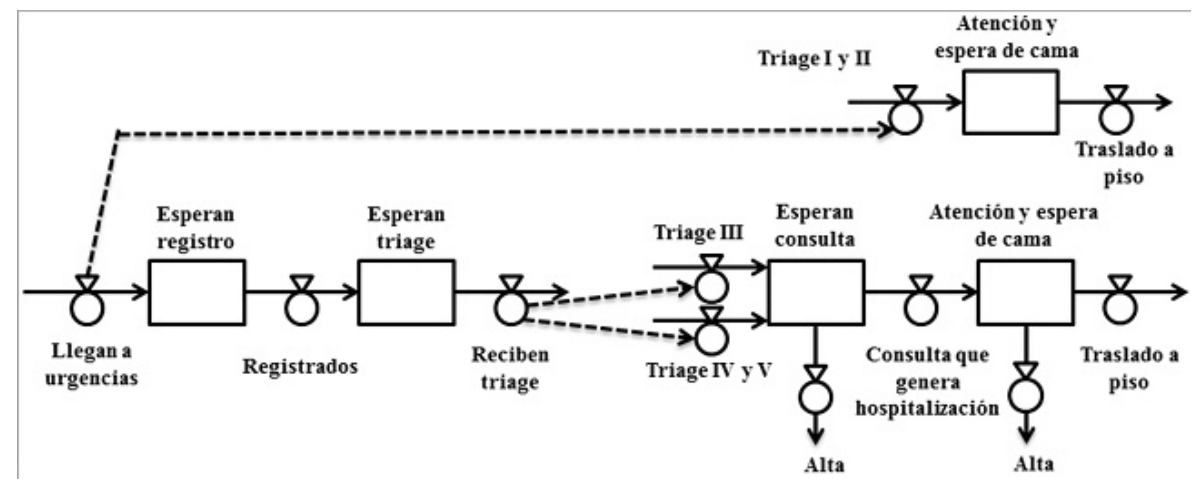

FIGURA 3

Unidad de urgencias típica

Fuente: elaboración propia.

Se definió un escenario base con la información histórica de un mes de operaciones de un hospital de nivel tres en la ciudad de Medellín, así: i) llegada: los datos de entrada corresponden al registro histórico de pacientes que arribaron al servicio; ii) registro: se simula la capacidad que tienen dos personas para atender en el registro a seis pacientes por hora cada una; iii) triage: se supone que hay una persona cuya capacidad de atención es de doce personas por hora. La proporción de personas por tipo de triage es $1 \%$ para triage I, $19 \%$ para el II, $51 \%$ para el III, $14 \%$ para el IV y $15 \%$ para el V. A los pacientes de los triages I y II se les hace 
un proceso de estabilización cuya duración aproximada es de tres horas. Los pacientes de los demás triages esperan una consulta que es atendida por tres médicos cuya capacidad de atención se define de tres por hora. El $85 \%$ del triage III requiere algún tipo de tratamiento que se asume tarda cuatro horas. El 5\% de estos pacientes tratados serán dados de alta, mientras que el resto esperará traslado a piso. La tasa de traslados a piso es de aproximadamente veinte pacientes por día. Los pacientes con triage I y II tienen prioridad en el traslado a piso y no tienen que hacer cola para el registro o el triage.

Se simularon las siguientes estrategias, a partir del escenario base: 1) reorganización del registro, que se obtiene en el modelo eliminando la etapa del registro, así que esta labor se hace mientras los pacientes esperan el triage o la consulta; 2) implementación del fast-track, que consiste en un proceso independiente para atender con mayor celeridad a los pacientes de los triages $\mathrm{IV}$ y $\mathrm{V}$ por un médico, de los tres disponibles para consulta prioritaria, cuya capacidad de atención es de seis pacientes por hora; 3 ) se redireccionan los pacientes del triage IV y V a otro nivel del sistema; 4) aumento del número de traslados a pisos, por ejemplo, en 10\%, $30 \%$ y $50 \%$. Luego de estas cuatro estrategias puras, se plantea una combinación de ellas, de modo que se generan siete escenarios adicionales.

\section{Resultados}

\section{Situación y estrategias en unidades de urgencias de Medellín}

Como resultado del trabajo, a continuación se describe la situación de cada una de las instituciones que participaron en el estudio. Se destaca el grado de saturación de cada una y las estrategias implementadas para afrontarla.

\section{Unidad 1 (hospitalpúblico)}

El servicio de urgencias médicas se divide en adultos, ginecobstétricas y pediátricas. Para la admisión cuenta con cuatro taquillas, tres consultorios para triage y seis para evaluación médica. Por el tamaño de su infraestructura permite contar con una disponibilidad de 52 camillas, 15 camas y 6 sillas. Dispone de salas de reanimación, procedimientos, pacientes aislados, suturas, observación, trabajo de parto y recuperación. Los corredores se usan como salas de expansión, donde se utilizan hasta 15 camillas y 20 sillas adicionales. La necesidad de atención es soportada por 18 médicos, distribuidos en diferentes turnos, 5 enfermeras en el día y 2 en la noche; también cuenta con urgentólogo, ortopedista, pediatra, ginecoobstetra y anestesiólogo.

En esta institución el servicio está organizado como se plantea en el esquema de la figura 1. Los usuarios con triage I ingresan por una entrada distinta para recibir atención inmediata, y los demás son recibidos por personal de seguridad y dirigidos a una sala de espera para el registro. En algunas ocasiones se recomienda a pacientes con niveles de gravedad leve trasladarse a unidades de menor complejidad. El usuario continúa al triage, donde se sigue la clasificación australiana (I a IV). El hospital tiene una promesa de atención de 0 , 30 y 120 minutos para los primeros tres niveles de triage, y un lapso más prolongado no especificado para los pacientes de triage IV. Posteriormente al triage, el paciente pasa a consulta, y luego a hospitalización, de ser necesario.

La saturación se presenta sobre todo durante la consulta, en la cual el proceso se congestiona con mayor frecuencia y magnitud. Frente a esto, la institución plantea las siguientes estrategias: destina más personal de otras áreas para atender las críticas dentro de urgencias; cuando el número de pacientes supera el número de camas y camillas, el corredor de observación es utilizado como área de expansión y se ubican más pacientes; también cuenta con un sistema conocido como alta temprana, soportado por otra institución. Otra estrategia importante es el hospital en casa, dirigido a pacientes de medicina general donde personal médico realiza 
visitas periódicas para evaluar la evolución de la enfermedad. La estrategia más significativa que permite atenuar la saturación es la realizada en la consulta, en la que se comparte personal asistencial priorizando en el triage III, que es en el cual se concentra la mayor población que inicia la búsqueda de los servicios de urgencias.

\section{Unidad 2 (hospital universitario)}

La institución es privada, sin ánimo de lucro y presta servicios de salud con énfasis en alta complejidad. Por su filosofía de atención y prestación de servicios destina grandes esfuerzos a quienes necesitan servicios y no poseen capacidad de pago, además de ser una institución que por su tradición posee la mayor demanda entre las unidades visitadas. Cuenta con cuatro consultorios médicos, un consultorio de ortopedia, 40 camillas y 48 camas, así como salas de reanimación, atención inmediata y atención de intoxicados. También tiene cuatro quirófanos apoyados con salas de recuperación, cuidados intensivos, observación, cuidados intermedios, yesos, hospitalización para pacientes quemados y prehospitalización. Durante la atención en urgencias hacen presencia diferentes especialistas y se cuenta con servicios de apoyo diagnóstico y terapéutico. Debido a la gran demanda, la unidad busca expandir los recursos, procurando brindar condiciones mínimas para la estancia.

El proceso de atención presenta una variación con respecto al esquema de la figura 1 , debido a que el registro se realiza en la consulta y no es requisito para iniciar el proceso. Los pacientes ingresan por la misma entrada. Dada la vocación de la institución, el vigilante no ejerce ningún control y las barreras de acceso para continuar en los servicios están determinadas por los tiempos de espera en cada nodo del flujo. El usuario continúa al triage, donde se sigue la clasificación Manchester ( $\mathrm{I}$ a V). Los pacientes urgentes (triage I) ingresan inmediatamente a las salas de reanimación, quirófanos o traumas, de acuerdo con su riesgo. El usuario que no requiere atención inmediata pasa directamente a triage, ya que se elimina el registro y este se realiza en la consulta. Posteriormente al triage, el usuario pasa por una sala de espera y continúa a la consulta o diagnóstico, para luego ser hospitalizado.

Esta institución es la que presenta mayor saturación entre las unidades visitadas, especialmente en la etapa del triage hacia la consulta, donde se concentran en mayor proporción los triages III, IV y V. Para reducir la saturación se desarrolla la estrategia fast-track, la cual está dirigida a los pacientes con lesiones o enfermedades leves, en general triages IV y V, que pueden acelerar el flujo de los pacientes y reducir problemas propiciados por los usuarios inconformes debido a la larga estancia en el servicio. A efectos de acelerar la atención de pacientes de triages II y III, se pasan a una sala prehospitalaria a la espera de cama. Igualmente, la institución puede derivar a los pacientes cuya lesión no es acorde con su nivel de complejidad, para lo cual tiene canal de comunicación con la red pública de primer nivel.

\section{Unidad 3 (clinica universitaria)}

La institución es mixta, sin ánimo de lucro y presta servicios de mediana y alta complejidad. En urgencias cuenta con 121 camas, de las cuales 98 son para adultos, cuatro para el área de pacientes críticos y 19 para urgencias pediátricas. Dispone de cuatro taquillas para admisión de adultos y una para niños, dos consultorios para triage y seis para consulta o evaluación por médico. Además, tiene ocho salas para tratamiento inicial, atención inmediata, traumas, pacientes críticos y observaciones. Por otra parte, la disponibilidad de recursos médicos es de un urgentólogo, un ortopedista, ocho médicos generales, dos jefes de enfermería y once auxiliares de enfermería. Debido a su estructura y a las estrategias que desarrolla para atenuar la saturación, no necesita realizar la expansión de recursos.

Esta institución es la que menos problemas de saturación presenta entre las visitadas. El proceso de gestión varía respecto al esquema de la figura 1 en la variación del triage por el registro. Todos los pacientes ingresan por el mismo lugar para ser clasificados en el triage, el cual sigue la clasificación Manchester (I a V), aunque los 
pacientes que requieren atención inmediata debido a su gravedad (triage I) no realizan este proceso. Después de ser clasificados, los pacientes pasan al registro de sus datos básicos y papeleo normativo. De continuar en el proceso, los pacientes pasan a consulta y permanecen en observación y, dependiendo del estado de su enfermedad, son dados de alta o se les asigna una cama en hospitalización.

La leve saturación que se presenta en el servicio se da en el triage, al clasificar los pacientes según nivel de gravedad, y en el registro de los usuarios. Las estrategias para atenuar la saturación están definidas en el cambio del flujo en el proceso de atención de los pacientes: primero se clasifica el riesgo y luego se registra, así el hospital evita realizar trámites innecesarios, dado que algunos pacientes después de ser clasificados en niveles IV y $\mathrm{V}$ son derivados a otras instituciones; en otras palabras, la estrategia de esta unidad consiste en redireccionar los triages IV y $\mathrm{V}$.

\section{Unidad 4 (clinica privada)}

La institución, para mejorar la gestión del proceso, realiza la independencia de los servicios, diferenciando la atención inmediata de pacientes críticos (emergencias) y las urgencias para las demás personas. Para las emergencias cuenta con un quirófano, dos salas de reanimación, una sala de ortopedia, una sala de toxicología; por su parte, las urgencias médicas cuentan con un área independiente para triage, observación de adultos con vientiocho cubículos, observación pediátrica con diez cubículos y ocho consultorios para atención prioritaria. La unidad posee los servicios de laboratorio, farmacia e imágenes diagnósticas las veinticuatro horas del día.

El proceso de gestión varía especialmente respecto al esquema de la figura 1 en la división de los servicios de emergencias y urgencias. El proceso de flujo en el interior de los servicios inicia cuando el paciente es recibido por un camillero y lo dirige a recepción para el registro; es importante para la institución revelar la entidad pagadora. De allí, puede ser direccionado a otra institución de menor complejidad o continúa la atención para ser clasificado por triage. La clasificación de triage que se sigue es la de Manchester (I a V). Si es triage I o II, va directamente a los servicios de emergencias médicas, mientras que triage III, IV y V se concentra en la unidad de urgencias para consulta inicial y posible hospitalización. Cada proceso de atención tiene un tiempo de espera según su clasificación y el tipo de aseguramiento. El vigilante, que normalmente recibiría al paciente en la entrada, es ubicado entre el triage y la atención inicial para realizar seguimiento al pago de los pacientes.

La reciente ampliación física de esta unidad ha llevado a que no se presenten problemas de saturación, aunque es posible identificar algunas estancias de tiempo sin mucha significancia del registro al triage. Las estrategias desarrolladas para mejorar la eficiencia de la institución principalmente radican en la división entre las emergencias y las urgencias médicas. Cuando se observa saturación en las urgencias se agiliza el triage, por medio del aumento de profesionales médicos y enfermeras, quienes apoyan la labor y hacen seguimiento al comportamiento de las camas hospitalarias con el fin de aumentar la productividad en función de su rotación. 
TABLA 2

Unidades, grado de saturación y estrategias

\begin{tabular}{|c|c|c|}
\hline Unidad & \begin{tabular}{c|} 
Grado de \\
saturación
\end{tabular} & Estrategias \\
\hline Hospital público & Alto & $\begin{array}{l}\text { - Altas hospitalarias } \\
\text { - Prioriza triage III } \\
\text { - Hospital en casa } \\
\text { - Altas tempranas triage IV }\end{array}$ \\
\hline $\begin{array}{l}\text { Hospital } \\
\text { universitario }\end{array}$ & Alto & $\begin{array}{l}\text { - Registro se realiza en consulta } \\
\text { - Consulta fast track para triage IV } \\
\text { - Envio a sala prehospitalaria }\end{array}$ \\
\hline $\begin{array}{l}\text { Clínica } \\
\text { universitaria }\end{array}$ & Medio & $\begin{array}{l}\text { - Varía triage por registro } \\
\text { - Registro en momento de espera } \\
\text { - Enfermeras realizan triage } \\
\text { - Admisión considerando la capacidad } \\
\text { - Redirección de triage IV y V }\end{array}$ \\
\hline Clínica privada & Bajo & $\begin{array}{l}\text { - Entrada diferente para emergencias y } \\
\text { urgencias } \\
\text { - Realiza traslados triage III, IV y V } \\
\text { - Agiliza triage por medio del aumento } \\
\text { de profesionales médicos y enfermeras. }\end{array}$ \\
\hline
\end{tabular}

Fuente: elaboración propia.

\section{Simulación de estrategias en hospital característico}

La simulación de las estrategias para atenuar la saturación en el hospital típico o característico parte del escenario base, el cual tiene un grado de saturación del 168\%, una estancia promedio de 22.03 horas (triage I 0.38 horas, triage IV y V 2.78 horas, y triage II y III 24.04 horas), 186 pacientes en el servicio al final del mes, entre los que usan cama y los que están sentados, 2034 pacientes trasladados a piso, y 1432 egresos de urgencias, para un total de 3466 pacientes atendidos en el mes. En la tabla 3 se presentan los resultados de cada estrategia.

La primera estrategia es una solución de flujo que consiste en reorganizar el registro como lo hace la unidad 3, de modo que este no se hace al ingreso sino en el curso de la atención. De este modo, se reduce la espera en el servicio. Como resultado de este movimiento, se presenta una reducción importante en la estancia de los pacientes de los triages IV y V, que pasa de 2.78 a 1.83 horas, y un poco en el caso de los pacientes de los triages II y III, de 24.04 a 22.72 horas. Con esta reducción, la estancia promedio pasa de 22.03 a 20.37 horas, y al final se presenta una leve disminución en el número de pacientes que permanecen en el servicio, así que hay una pequeña contribución a la reducción de la saturación (3.8\%) y un tiempo menor en todo el servicio (8\%). 
La segunda estrategia de flujo es la implementación del fast track, desarrollado por la unidad 2. En este caso no se aprecian cambios importantes en el servicio. Como resultado, solo se alcanza una leve reducción en la estancia de los pacientes de triage IV y V.

La tercera estrategia también es de flujo y consiste en la remisión de los pacientes de los triages IV y V a otro nivel de menor complejidad, como lo hace la unidad 1, así que los pacientes son admitidos y pasan por triage, pero una vez son clasificados en estos niveles no continúan en el servicio. En este caso, los resultados muestran la reducción total en la estancia de los pacientes de triage $\mathrm{IV}$ y V, que pasa de 2.78 a 0 horas, y una leve reducción en el caso de los pacientes de triage II y III. Con esta reducción, la estancia promedio pasa de 22.03 a 21.33 horas. Así, hay una pequeña contribución a la reducción de la saturación del servicio y un menor tiempo en todo el servicio.

La cuarta estrategia es una solución de salida, basada en el incremento de traslados a pisos como lo desarrolla la unidad 4. En la medida en que hay un mayor número de traslados, se produce una reducción del tiempo en el servicio, especialmente para los pacientes de los triages II y III, se reducen la ocupación y el número de personas que permanecen al final, y se da un ligero aumento en el número de atendidos. Para reducir la ocupación por debajo del $100 \%$ es necesario aumentar el traslado a pisos en un $50 \%$, en cuyo escenario la estancia promedio pasa de 22.03 a 16.91 horas, y los pacientes al final del periodo se reducen en cerca del $38 \%$ (véase figura 4 ).

TABLA 3

Tabla de resultados

\begin{tabular}{|c|c|c|c|c|c|c|c|}
\hline N. ${ }^{\circ}$ & Estrategias & $\begin{array}{l}\text { Ocupación } \\
\text { servicio }\end{array}$ & $\begin{array}{c}\text { Tiempo } \\
\text { todos los } \\
\text { triages (h) }\end{array}$ & $\begin{array}{c}\text { Personas al } \\
\text { final de } \\
\text { urgencias }\end{array}$ & $\begin{array}{l}\text { Traslados a } \\
\text { piso } \\
\text { (pacientes/ } \\
\text { mes) }\end{array}$ & $\begin{array}{c}\text { Egresos } \\
\text { (pacientes/ } \\
\text { mes) }\end{array}$ & $\begin{array}{c}\text { Total } \\
\text { atendidos } \\
\text { (pacientes / } \\
\text { mes) }\end{array}$ \\
\hline $\mathbf{0}$ & Escenario base & 168 & 22.03 & 186 & 2034 & 1432 & 3466 \\
\hline & \multicolumn{7}{|c|}{ Estrategias puras } \\
\hline 1 & $\begin{array}{l}\text { 1. Reorganizar registro } \\
\text { (flujo) }\end{array}$ & 168 & 20.37 & 179 & 2038 & 1435 & 3473 \\
\hline 2 & $\begin{array}{l}\text { 2. Implementar fast- } \\
\text { track (flujo) }\end{array}$ & 168 & 22 & 186 & 2034 & 1432 & 3466 \\
\hline 3 & $\begin{array}{l}\text { Remisión de los } \\
\text { triages IV y V a otro } \\
\text { nivel del sistema } \\
\text { (flujo/entrada) }\end{array}$ & 167 & 21.33 & 183 & 2034 & 378 & 2412 \\
\hline \multirow{6}{*}{4} & $\begin{array}{l}\text { Incrementar traslados } \\
\text { a pisos }(10 \%) \text { (salida) }\end{array}$ & 136 & 20,2 & 154 & 2066 & 1432 & 3498 \\
\hline & \begin{tabular}{|l|} 
Incrementar traslados \\
a pisos (30\%)
\end{tabular} & \multirow[t]{2}{*}{117} & \multirow[t]{2}{*}{18} & \multirow[t]{2}{*}{135} & \multirow[t]{2}{*}{2085} & \multirow[t]{2}{*}{1432} & \multirow[t]{2}{*}{3517} \\
\hline & (salida) & & & & & & \\
\hline & $\begin{array}{l}\text { Incrementar traslados } \\
\text { a pisos }(50 \%)\end{array}$ & \multirow[t]{2}{*}{98} & \multirow[t]{2}{*}{16.91} & \multirow[t]{2}{*}{116} & \multirow[t]{2}{*}{2104} & \multirow[t]{2}{*}{1432} & \multirow[t]{2}{*}{3536} \\
\hline & (salida) & & & & & & \\
\hline & \multicolumn{7}{|c|}{ Estrategias mixtas } \\
\hline 5 & 1 "+" 2 & 168 & 20.35 & 179 & 2038 & 1435 & 3473 \\
\hline 6 & $1 "+" 3$ & 168 & 19.72 & 176 & 2038 & 379 & 2417 \\
\hline 7 & $1 "+" 4-30 \%$ & 118 & 16.28 & 129 & 2088 & 1435 & 3523 \\
\hline 8 & $2 "+" 4-30 \%$ & 117 & 17.99 & 135 & 2085 & 1432 & 3517 \\
\hline 9 & $3 "+" 4-30 \%$ & 117 & 17.34 & 133 & 2085 & 378 & 2463 \\
\hline 10 & $3 "+" 4-30 \%$ & 118 & 16.27 & 129 & 2088 & 1435 & 3523 \\
\hline 11 & $1 "+" 3 "+" 4-30 \%$ & 118 & 16.27 & 126 & 2088 & 379 & 2467 \\
\hline
\end{tabular}


En las estrategias mixtas, aunque no hay cambios muy significativos, se destaca la número 6 al combinar la reorganización del registro con la remisión de los triages IV y V. En este caso, la ocupación no varía, pero se produce una mayor reducción en los tiempos de espera. Por lo demás, los resultados siempre van a estar influenciados por lo que significa el aumento del traslado a pisos, que se unificó para ilustrarlo en el $30 \%$ (véase tabla 3); se nota que los tiempos de espera tienden a bajar más cuando se combina con la reorganización del registro.

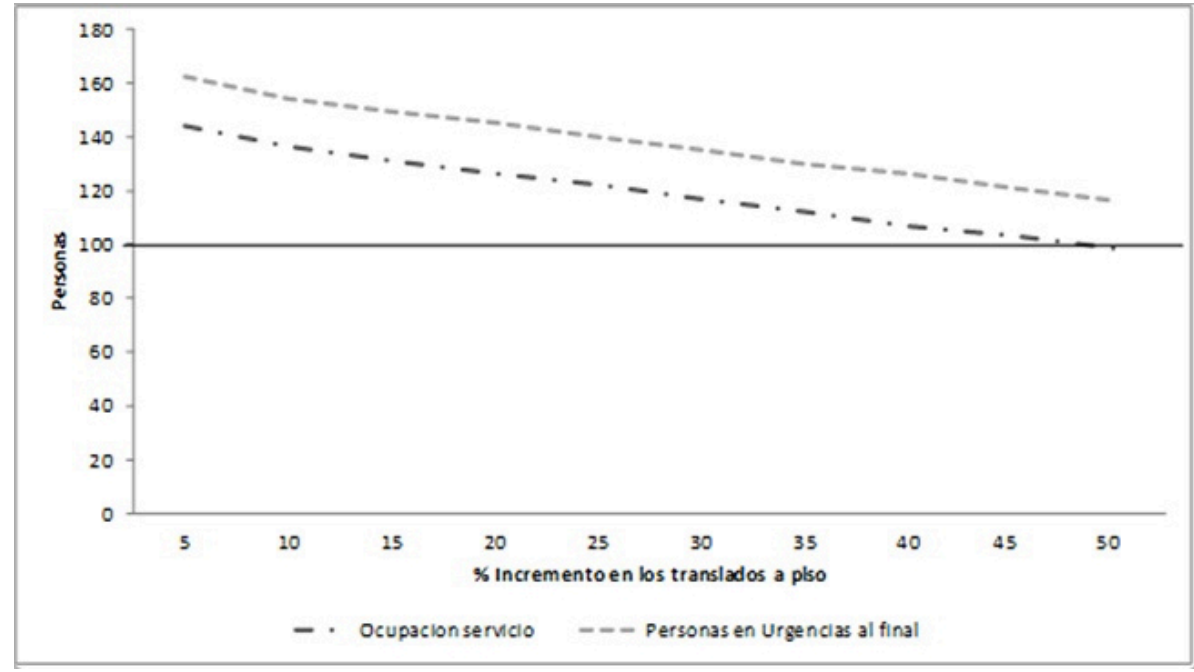

FIGURA 4

Reducción de la ocupación en los servicios de urgencias debido a la estrategia 4 Fuente: elaboración propia.

\section{Discusión}

La demanda por los servicios de urgencias médicas en las unidades estudiadas tiene una forma similar a la que se registra en la literatura, así que su composición da cuenta de una baja participación de los dos primeros triages y una concentración en el triage tres, seguido de los últimos triages que también representan una proporción importante. Esto indica que la demanda se concentra en atenciones menos graves y las emergencias médicas significan una baja proporción.

La demanda se encuentra además influenciada por el tipo de institución y por el perfil de la población que acude a ellas, de modo que tiende a ser más alta para las unidades de carácter público o que debido a su misión no aplican barreras a la entrada, lo que facilita la búsqueda de atención por parte de personas de más bajos recursos. En cambio, en instituciones privadas con fin de lucro o con barreras a la entrada, la demanda es menor y tiene una mayor participación la población de estratos medios y altos, incluyendo usuarios de planes de medicina prepagada a los que se suele dar prelación.

La saturación, medida tanto por la ocupación como por los tiempos de atención [8], resulta explicada por factores externos a los hospitales, principalmente los flujos de entrada que están determinados por fenómenos epidemiológicos y causas externas que no son controlables por las unidades de urgencias. Sin embargo, como se plantea en el trabajo de Asplin [4], las soluciones para influenciar la demanda parecen imposibles, excepto que se puedan impactar los factores de riesgo o que el sistema de salud ofrezca oportunidad y calidad en la atención de los servicios ambulatorios. En las unidades de urgencias más afectadas por la saturación y por la presión que ejerce la demanda de los triages superiores, se mencionó mucho esta situación, que se origina a partir de la falta de oportunidad en consulta médica o de la discontinuidad en tratamientos de enfermedades crónicas. 
Aunque usualmente se asume que los servicios de urgencias se saturan por factores externos, lo cual indica una demanda superior a la disponibilidad, en todo caso poco se aborda el potencial de los hospitales para mejorar su eficiencia. Es así como el estudio ofrece un abordaje sobre la capacidad de gestión de los servicios y las estrategias para reducir la ocupación y los tiempos de atención. Las simulaciones efectuadas muestran cómo las estrategias que implementan medidas para aumentar el flujo del paciente, garantizando el traslado a pisos, tienen un mejor desempeño que aquellas que reorganizan el proceso o aumentan la capacidad de atención.

En términos de ocupación, las estrategias de entrada y flujo no tienen un impacto significativo, aunque reducen los tiempos promedio en urgencias en todos los triages. Por esto, es necesario desarrollar estrategias de salida, en lo cual la unidad de urgencias no posee autonomía pues depende de la disponibilidad de camas en el hospital o en la red de servicios. Esto comprende una utilización eficiente de recursos, representada en el número de camas y la capacidad de seguimiento a su rotación, pues a la salida de los servicios de urgencias se está generando el cuello de botella que los satura.

El triage es una de las etapas más sensibles para reducir tiempos de estancia en los servicios, por lo que se identifican diferencias entre los triages utilizados por las unidades de urgencias visitadas. En ese sentido, el triage también despierta gran interés debido a que en la literatura se menciona que esta etapa es realizada por personal de enfermería, pero en las unidades visitadas este proceso lo lleva a cabo un profesional médico, muchas veces apoyado por enfermeras. El triage como evaluación y clasificación de la gravedad parece estar sometido a mayor rigor según la disponibilidad de camas; esto es, a mayor ocupación de la capacidad instalada se puede tender a clasificar de manera más estricta la urgencia médica y en tal sentido se reduce el número de pacientes admitidos para ser atendidos en el servicio, y en caso contrario se puede dar lugar a una mayor flexibilidad dado que habría mayor disponibilidad. También existe la posibilidad de diferenciar los niveles de triage, pero condicionado por la infraestructura del hospital, de modo que los usuarios "emergentes", generalmente de triage I, ingresan por una entrada diferente al resto de pacientes, lo que mejora la capacidad de reacción para ellos, y el espacio destinado para los usuarios de un nivel de triage de menor complejidad.

\section{Conclusiones}

Los servicios de urgencias médicas son una expresión de la capacidad de resolución que tiene el sistema de salud. Sin duda, el hecho de que haya saturación da cuenta de una brecha entre la demanda o las necesidades y la oferta o disponibilidad de recursos. Sin embargo, la solución no puede ser, o no debería ser exclusivamente, la de ampliar la capacidad de los servicios de urgencias, pues esto ocasiona mayores costos y no se estaría reconociendo las verdaderas causas.

Las soluciones deben ser integrales, dentro de la concepción de una red de servicios y de garantizar el acceso integral, de manera que se mantenga una oferta de urgencias más destinada a atender emergencias médicas, bien articulada con la red hospitalaria para permitir un flujo adecuado de los pacientes conforme a los tiempos de atención esperados. En cambio, corresponde al sistema de salud y a las políticas de otros sectores controlar los riesgos y atender la demanda oportunamente mediante servicios ambulatorios, incluyendo la opción que ofrecen varias entidades de atenciones prioritarias, pero mediante una infraestructura blanda, que no implica grandes costos.

Al comparar los resultados del escenario base con relación a las estrategias puras de entrada, flujo y salida, más la combinación entre estas, se puede apreciar que todas las estrategias reducen la estancia en urgencias, pero solo las estrategias de salida y las que se combinan con ella tienen efectos significativos sobre la ocupación, pues las estrategias de entrada y flujo y su combinación no tienen efecto sobre el grado de ocupación. Respecto a la estrategia de aumentar el traslado a pisos, esta permite mejorar la ocupación, pues cuando se aumentan los traslados a piso en un $10 \%$ la ocupación se reduce en un $19 \%$, mientras si se aumentan los traslados a 
$30 \%$ y $50 \%$, se alcanza una reducción de la ocupación de 30.4\% y 41.7\%, respectivamente. En este sentido, las estrategias mixtas $8,9,10$ y 11 que se combinan con el traslado a piso en un margen del $30 \%$ logran reducir la ocupación en un intervalo entre $30.4 \%$ y el $29.8 \%$.

Por otro lado, todas las estrategias no afectan de manera significativa el aumento de los egresos, pues la estrategia de reorganizar el registro y las estrategias mixtas 6, 8 y 10 tienen leves incrementos, las demás estrategias mantienen constantes o reducen la cantidad de egreso, debido a que los pacientes atendidos en urgencias continúan en el interior del servicio, usando una camilla o sentados dentro de los servicios de urgencias. Respecto al total de personas atendidas por mes, la estrategia de fast-track se mantiene constante con relación al escenario base, y la estrategia de redireccionar los triage $\mathrm{IV}$ y $\mathrm{V}$ presta servicios a menor número de personas. Todas las estrategias mixtas que se combinen con esta estrategia no tendrán efecto sobre el total de atendidos.

Finalmente, entre mayor sea el porcentaje de traslados a piso, es decir, tener un mayor número de camas y control de la circulación de pacientes, se puede afectar considerablemente la saturación, la reducción de estancia, el aumento de egresos y el incremento de pacientes atendidos. Si se logra realizar un traslado a piso del 50\%, no solo se reduce el tiempo total de atención a 16.91 horas, sino que se reduce la ocupación del servicio de urgencias a un 98\%, mientras que las personas al final del mes serían 116, los egresos de pacientes continuarían en 1432 y el total de pacientes atendidos en el mes aumentarían en 3536.

A partir de esta investigación es posible desarrollar un modelo de vigilancia sobre el acceso a los servicios de urgencias. No obstante, dado que no fue posible reunir información de todas las instituciones, es importante realizar actividades de aproximación y de sensibilización con las instituciones, a fin de comprometerlas con un mejor registro de información, su entrega o reporte oportuno y la apertura para realizar o gestionar mejoras que permitan mayores facilidades para el acceso que conlleven reducir la saturación.

Es así como la Alcaldía de Medellín ha desarrollado estrategias para reducir la saturación de los servicios de urgencias, especialmente en las unidades de mayor complejidad. Para ello, se han desarrollado mesas de trabajo conformadas en la Comisión de Urgencias de la Secretaría de Salud, el Centro Regulador de Urgencias y el Centro Integrado de Gestión al Acceso en Salud (CIGA). Estos programas han desarrollado soluciones de entrada o flujo, pues las estrategias que se han generado o promovido son el aumento de la oferta de atención médica en las EPS, aumento de su calidad y remisión de pacientes con bajos niveles de riesgo (triages IV y V). El programa (CIGA), al integrar un centro de referencia, ha permitido gestionar de manera oportuna citas médicas para aquellos pacientes que van a urgencias, pero cuya situación de salud no representa un riesgo para su vida y no requieren una atención inmediata, tal como lo realiza la clínica universitaria. Con este tipo de medidas se busca reducir la saturación de las urgencias en un 30\% en 2017.

\section{Agradecimientos}

Los autores agradecen a las personas y a las instituciones que colaboraron brindando información y aportando sus opiniones para que este estudio fuera posible, así como la financiación por parte de la Universidad de Antioquia y de la Secretaría de Salud de Medellín. Se agradece especialmente a los integrantes de la Comisión Salud de Medellín y a los profesionales de la Secretaría de Salud y de los hospitales que decidieron participar en el estudio. El artículo de investigación derivado del proyecto Acceso a los servicios de salud en la ciudad de Medellín: caracterización y propuesta para el monitoreo y la vigilancia, cuya fase correspondiente a la formulación de un modelo de vigilancia del acceso a servicios de urgencias médicas fue llevada a cabo entre el 17 de marzo y el 17 de septiembre de 2014. Financiación: Secretaría de Salud de Medellín y Universidad de Antioquia (Convenio de Asociación 4600047513 de 2013). 


\section{Referencias}

1. Restrepo JH. Economía y financiamiento de la salud. En: Maya JM, Blanco JH, editores. Fundamentos de salud pública, tercera edición. Medellín: CIB; 2014.

2. Crawford K, Morphet J, Jones T, Innes K, Griffiths D, Williams A. Initiatives to reduce overcrowding and access block in Australian emergency departments: A literature review. Collegian. 2014; 21(4):359-66.

3. Obermeyer Z, Abujaber S, Makar M, Stoll S, Kayden S, Wallis L, Reynolds T, on behalf of the Acute Care Development Consortium. Emergency care in 59 low- and middle-income countries: a systematic review. Bulletin of the World Health Organization 2015 May; (93):577-86.

4. Asplin BR, Magid DJ, Rhodes KV, Solberg LI, Lurie N, Camargo CA. A conceptual model of emergency department crowding. Annals of Emergency Medicine. 2003; 42(2):173-80.

5. Paul SA, Reddy MC, DeFlitch CJ. A systematic review of simulation studies investigating emergency department overcrowding. Simulation. 2010; 86(8-9):559-71.

6. GES, Grupo de Economía de la Salud. Acceso a servicios de salud: Panorama colombiano, medición y perspectiva de actores en Medellín. Observatorio de la Seguridad Social. 2014; (28).

7. Boyle A, Beniuk K, Higginson I, Atkinson P. Emergency department crowding: time for interventions and policy evaluations. Emergency Medicine International. 2012 feb 7; 2012. doi: 10.1155/2012/838610.

8. Oredsson S, Jonsson H, Rognes J, Lind L, Goransson KE, Ehrenberg A, et al. A systematic review of triage-related interventions to improve patient flow in emergency departments. Scand J Trauma Resusc Emerg Med. 2011; 19(1).

9. Kataoka, SH, Zhang L, Wells KB. Unmet need for mental health care among US children: Variation by ethnicity and insurance status. American Journal of Psychiatry. 2002; 159(9):1548-55.

10. Velásquez P, Rodríguez A, Jaén J. Metodologías cuantitativas para la optimización del servicio de urgencias: una revisión de la literatura. Revista Gerencia Políticas de Salud. 2011; 10(21):196-218.

11. Roberge D, Pineault R, Larouche D, Poirier LR. The continuing saga of emergency room overcrowding: are we aiming at the right target? Healthcare Policy. 2010; 5(3):27-39.

12. Khangura JK, Flodgren G, Perera R, Rowe BH, Shepperd S. Primary care professionals providing non-urgent care in hospital emergency departments. Cochrane Database of Systematic Reviews. 2012; Issue 11. Art. No.: CD002097.

13. Villa-Roel C, Guo X, Holroyd BR, Innes G, Wong L, Ospina M, et al. The role of full capacity protocols on mitigating overcrowding in EDs. The American Journal of Emergency Medicine. 2012; 30(3):412-20.

14. Saturación en servicio de urgencias. El Diario del Otún. 2013, 3 de mar. Disponible en: http://www.eldiario.com. $\mathrm{co} /$ seccion $/$ RISARALDA $/$ saturaci-n-en-servicio-de-urgencias $1303 . \mathrm{html}$ ?score $=4 \& \mathrm{id}=92973$

15. Baja, pero no se alivia saturación de urgencias. El Colombiano, 10 de junio de 2014. Disponible en: http://www.e lcolombiano.com/baja_pero_no_se_alivia_saturacion_de_urgencias-NXEC_298225

16. Rodríguez L. Propuestas para resolver saturación de servicios de urgencias en Medellín. Periodico El Pulso, Sección Generales. 2014; (190). Disponible en: http://www.periodicoelpulso.com/html/1407jul/general/general-16.h tm

17. ¿Qué está pasando con la salud en Villavicencio? El Tiempo, 26 de noviembre de 2014. Disponible en: http://ww w.eltiempo.com/colombia/otras-ciudades/panorama-de-la-salud-de-villavicencio/14891437

18. Martínez R. Si EPS cumplen, bajaría el colapso en las urgencias. El Colombiano, 10 de abril de 2015. Disponible en: http://www.elcolombiano.com/antioquia/si-eps-cumplen-bajaria-el-colapso-en-las-urgencias-NG1686395

19. Australasian College for Emergency Medicine. A National Triage Scale for Australian Emergency Departments [position paper]. Melbourne, Victoria, Australia: Australasian College for Emergency Medicine; 1993.

20. Cronin JG. The introduction of the Manchester triage scale to an emergency department in the Republic of Ireland. Accident and Emergency Nursing. 2003; 11(2):121-5. 
21. Grant MJ, Booth A. A typology of reviews: an analysis of 14 review types and associated methodologies. Health Information \& Libraries Journal, 2009; 26(2):91-108.

22. Forrester J. Industrial dynamics. Nueva York: MIT Press; 1961.

23. Castro JA, Cohen E, Lineros A, Sánchez R. Escala NEDOCS para medir congestión en urgencias: Estudio de validación en Colombia [internet]. Universidad del Rosario. Disponible en: http://hdl.handle.net/10336/2250

24. Rowe BH. Villa-Roel C, Guo X, Bullard MJ, Ospina M, Vandermeer B, et al. The role of triage nurse ordering on mitigating overcrowding in emergency departments: A systematic review. Academic Emergency Medicine. 2011; $18(12): 1349-57$.

\section{Anexo}

A continuación se detallan las ecuaciones del proceso:

- $\quad$ Llegan a urgencias $=\mathrm{GRAPH}(\mathrm{t}, \Delta \mathrm{t}$, pacientes que llegan $)$

- Registrados $=$ MIN (esperan registro $\Delta t$, funcionarios $x$ capacidad de atención hora)

- Reciben triage $=$ MIN (esperan triage $\Delta t$, personal asistencial $\mathrm{x}$ capacidad de atención hora)

- Triage I y II = llegan a urgencias $(\mathrm{p} 1+\mathrm{p} 1)$, donde $\mathrm{p} 1$ y $\mathrm{p} 2$ son las proporciones de pacientes con triages I y II.

- $\quad$ Triage III $=$ reciben triage $(\mathrm{p} 3)$, donde $\mathrm{p} 3$ es la proporción de pacientes con triage III.

- Triage IV y V = reciben triage $(\mathrm{p} 4+\mathrm{p} 5)$, donde $\mathrm{p} 4$ y $\mathrm{p} 5$ son las proporciones de pacientes con triage IV y V.

- Traslado a piso desde triage I y II = MIN (atención y espera de cama $\Delta \mathrm{t}$ camas disponibles en piso)

- Alta de consulta $=$ MIN (espera consulta $\Delta t$, personal asistencial x capacidad de atención hora) p6, donde $\mathrm{p} 6$ es la proporción de pacientes que son dados de alta.

- Consulta que genera hospitalización $=\operatorname{MIN}$ (espera consulta $\Delta t$, personal asistencial $\mathrm{x}$ capacidad de atención hora) (1-p6)

- Alta de atención y espera de cama = atención y espera de cama p7 / E, donde p7 corresponde a la proporción de personas que son atendidas y dadas de alta desde urgencias. E corresponde a la estancia promedio de la atención.

- Traslado a piso desde triage III = MIN (atención y espera de cama (1-p7) / E, camas disponibles en piso).

\section{Notas}

* Artículo de investigación.

1 Inicialmente se invitó a las clínicas y hospitales de mediana y alta complejidad (15 IPS) que conforman la denominada Comisión Salud. En esta instancia se esperaba compartir experiencias del modelo de vigilancia en las unidades de urgencias, conocer el comportamiento del flujo de información y propiciar espacios adecuados para analizar las estrategias que permitan reducir la saturación. Cuatro unidades aceptaron participar en el estudio.

\section{Licencia Creative Commons CC BY 4.0}

Como citar este artículo: Restrepo-Zea JH, Jaén-Posada JS, Espinal Piedrahita JJ, Zapata Flórez PA. Saturación en los servicios de urgencias: análisis de cuatro hospitales de Medellín y simulación de estrategias. Rev Gerenc Polít Salud. 2017; 17(34): 1-17. https://doi.org/10.11144/Javeriana.rgps17-34.ssua 